Computational

Mathematics

Division

The Growth Surface for the Slopes at the Boundary of a Polygon

J. Bernal

February 1994

\author{
Technology Administration \\ U.S. DEPARTMENT OF COMMERCE \\ National Institute of Standards and Technology \\ Gaithersburg, MD 20899
}

$Q C$

100

.056

\#53444 



\section{The Growth Surface for the Slopes at the Boundary of a Polygon}

\section{J. Bernal}

U.S. DEPARTMENT OF COMMERCE

Technology Administration

National Institute of Standards

and Technology

Applied and Computational Mathematics Division Computing and Applied Mathematics Laboratory Gaithersburg, MD 20899

February 1994

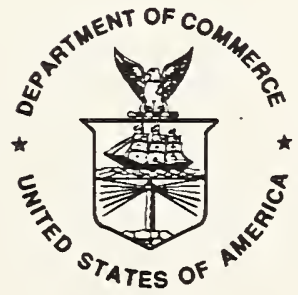

U.S. DEPARTMENT OF COMMERCE Ronald H. Brown, Secretary

TECHNOLOGY ADMINISTRATION

Mary L Good, Under Secretary for Technology

NATIONAL INSTITUTE OF STANDARDS

AND TECHNOLOGY

Arati Prabhakar, Director 



\title{
The growth surface for the slopes at the boundary of a polygon
}

\author{
Javier Bernal \\ National Institute of Standards and Technology, Gaithersburg, MD 20899.
}

\begin{abstract}
In this paper, we describe the growth surface for the slopes at the boundary of a polygon, and present a brute force algorithm for computing it.

Key words. Computational geometry, growth surface, medial axis, multiply-connected polygon, simple polygon, Voronoi diagram
\end{abstract}

AMS(MOS) subject classifications. $68 \mathrm{U} 05$ 


\section{Introduction}

Given $G$, a polygon in the plane, the Voronoi diagram for $G$ is a partition of the polygon into regions, each of which consists of the set of points in the polygon that are closer to a particular boundary edge or vertex of $G$ than to any other boundary edge or vertex. The union of the boundaries of these regions in the interior of $G$ contains what is called the medial axis of $G$ which is the set of points in the interior of $G$ that have at least two closest points on the boundary of $G . O\left(n^{2}\right)$ and $O(n \log n)$ algorithms for computing the Voronoi diagram for an arbitrary simple polygon with $n$ vertices, and therefore the medial axis of the polygon, have been presented in [2] and [1], respectively. Figure 1 illustrates the Voronoi diagram for a simple polygon. Figure 2 illustrates the medial axis of the same polygon.

The union of the boundaries of the regions in the Voronoi diagram for a polygon is a tree-like planar structure whose edges are straight-line segments and possibly portions of parabolic curves. It can be viewed as the points at which the regions meet as they are allowed to grow simultaneously from their edges or vertices at the same rate. Here two points in the polygon are encountered simultaneously during this growth process if and only if the distances from them to their corresponding edges or vertices are equal. In this paper, we present a new type of growth diagram for a polygon from which a tree-like planar structure can be extracted whose edges are all straight-line segments. In this model, it is assumed that the polygon is contained in the $x-y$ plane of 3 -dimensional space, and that a plane in 3-dimensional space has been assigned to each edge in the boundary of the polygon in such a way that given an edge the plane assigned to the edge contains the edge, the plane is not perpendicular to the $x-y$ plane, and an open set in the relative interior of the polygon whose closure includes the edge is contained in the lower open half-space defined by the plane. Under these assumptions and restricting the planes to the closed half-space above the $x-y$ plane, the polygon can be viewed as being enclosed by slopes that slant toward it, each of which originates in a particular edge in the boundary of the polygon. A growth diagram for the polygon can then be computed which is the perpendicular projection onto the polygon of a continuous 2-dimensional surface which we call the growth surface for the slopes at the boundary of the polygon (the growth surface for the polygon for short) and which is obtained by allowing regions to grow simultaneously from the edges of the boundary of the polygon at the same ascending rate along the slopes, each region growing where it is not impeded by other regions. Here two points in the polygon are encountered simultaneously during this growth process if and only if they are perpendicular projections onto the polygon of points in the growth surface whose $z$-coordinates are equal. The growth diagram for the polygon computed in this fashion is again a partition of the polygon into regions, each of which corresponds to a particular boundary edge of the polygon, and the boundaries of which 


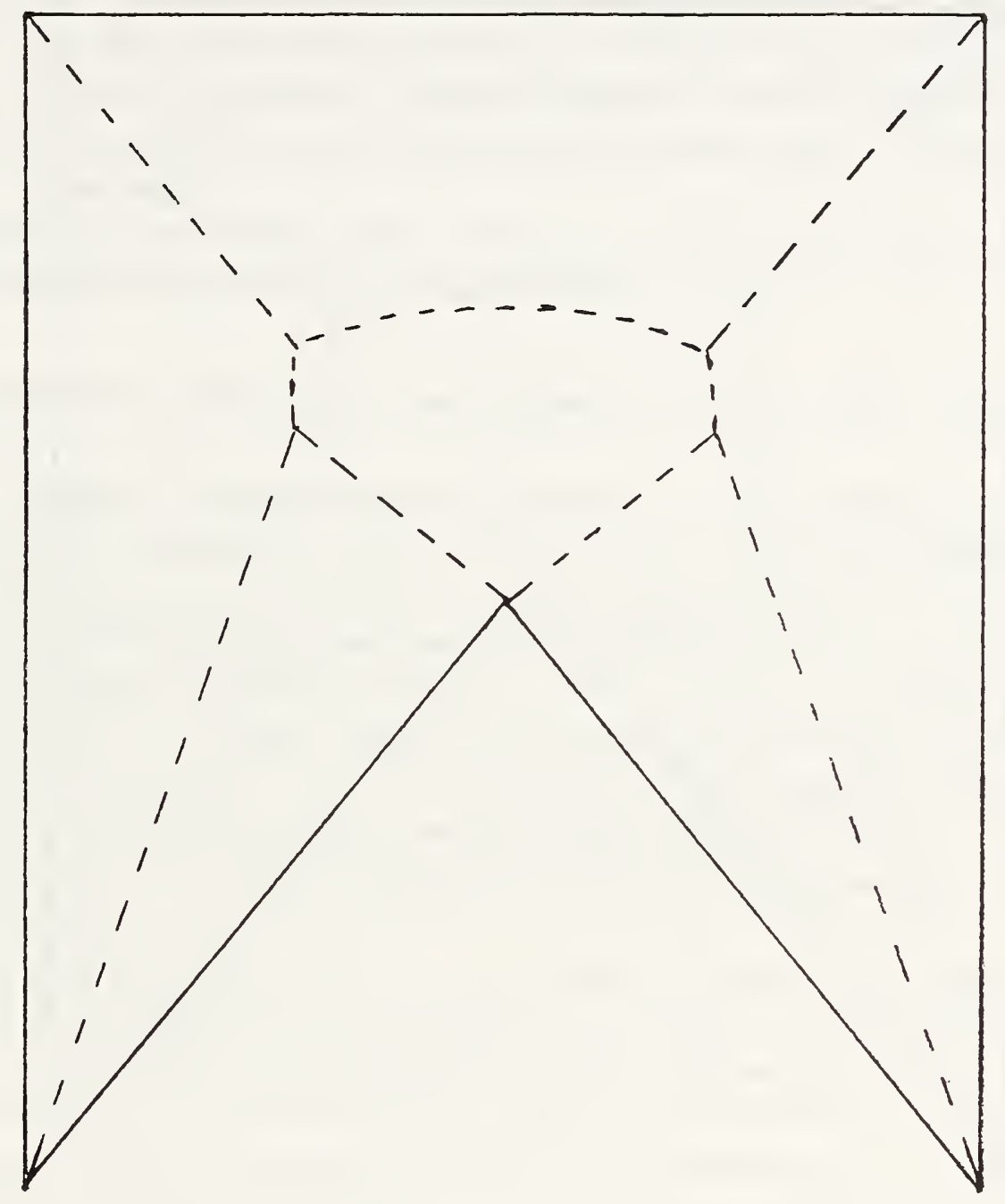

Figure 1: The Voronoi diagram for a simple polygon. 


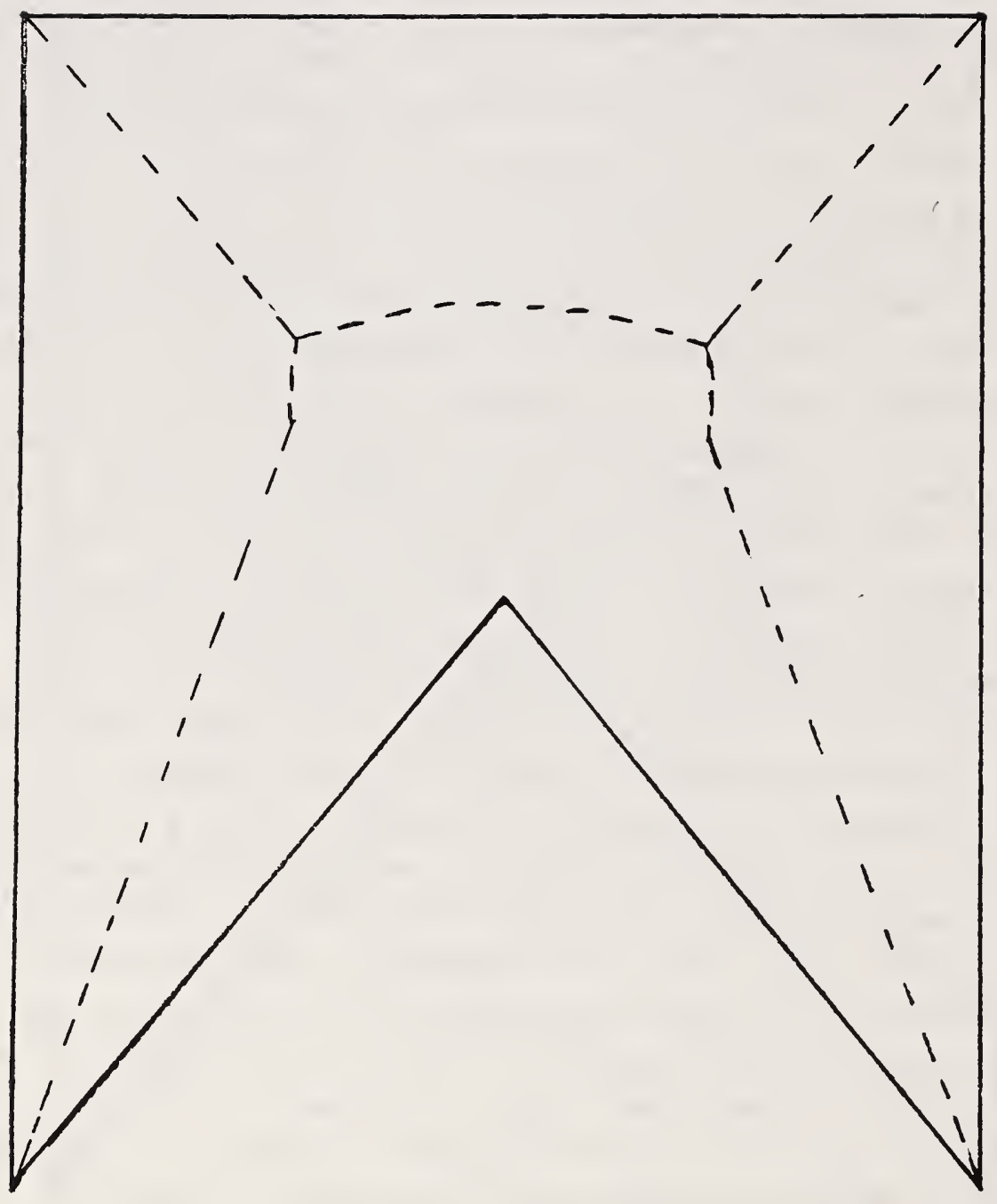

Figure 2: The medial axis of a simple polygon. 
consist of straight-line segments. Accordingly, a tree-like planar structure can be extracted from this diagram which is the union of the boundaries of the regions of the diagram in the relative interior of the polygon. This structure can also be viewed as the perpendicular projection onto the polygon of the ridge of the growth surface for the polygon, which is the set of points in 3-dimensional space where the regions of this surface meet as they grow in the manner previously described. Figure 3 illustrates the perpendicular projection onto the $x-y$ plane of the growth surface for a simple polygon when all slopes slant toward the polygon at the same angle.

In this paper, we describe the growth surface for a simple or multiply-connected polygon, and present a brute force algorithm for computing it.

\section{The growth surface for the slopes at the boundary of a polygon}

We consider a simple or multiply-connected polygon $G$ with $n$ vertices that is contained in the $x-y$ plane of 3 -dimensional space. We let $e_{i}, i=1, \ldots, n$, be the edges of the boundary of $G$. For each $i, i=1, \ldots, n$, we let $O_{i}$ be an open convex set in the relative interior of $G$ whose closure includes $e_{i}$, and we assume that planes $h_{i}, i=1, \ldots, n$, in 3 -dimensional space have been chosen such that for each $i, i=1, \ldots, n, e_{i}$ is contained in $h_{i}, h_{i}$ is not perpendicular to the $x-y$ plane, and $O_{i}$ is contained in the lower open half-space defined by $h_{i}$. In addition, for each $i, i=1, \ldots, n$, we let $\hat{h}_{i}$ be the closed half-plane in $h_{i}$ which is the intersection of $h_{i}$ and the upper closed half-space defined by the $x-y$ plane. With this notation, $G$ can then be viewed as being enclosed by the half-planes $\hat{h}_{i}, i=1, \ldots, n$, each of which slants toward it.

We suppose that for each $i, i=1, \ldots, n$, a region $R_{i}$ grows from edge $e_{i}$ along the halfplane $\hat{h}_{i}$ in such a way that all such regions grow simultaneously at the same ascending rate, each region growing where it is not impeded by other regions. Here the ascending rate is the rate at which the z-coordinates of points in a region change as the region grows, which without any loss of generality can be assumed to equal some undetermined positive constant. Under these assumptions, the problem considered is that of computing the topological structure of the continuous surface consisting of the regions $R_{i}, i=1, \ldots, n$.

We now make more precise the definition of the growth surface for the slopes at the boundary of the polygon $G$. Without any loss of generality we assume that $G$ is simple. We define the surface inductively, basing the induction on an integer variable $k$, which corresponds to special elevation levels that the surface achieves as it grows. The variable $k$ is set to zero at the start of the growth process and for some positive integer $q$ it is incremented by one $q$ times during this process. 


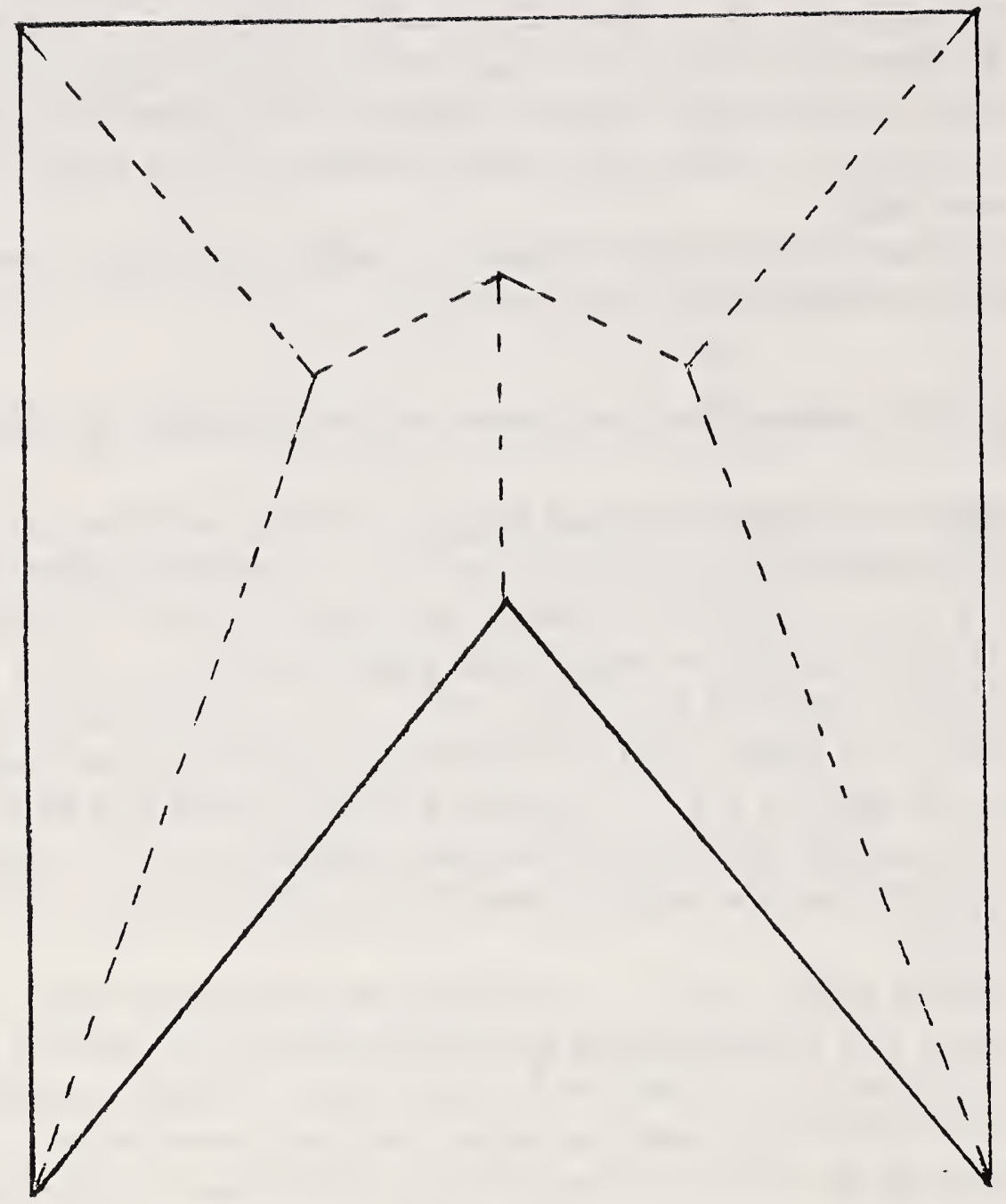

Figure 3: Perpendicular projection onto $x-y$ plane of growth surface for a simple polygon when all slopes slant toward the polygon at the same angle. 
The definition of the surface by induction proceeds as follows. First, we set $z_{0}=0, p_{0}=1$, $G_{10}=G, R_{i 0}=e_{i}, i=1, \ldots, n$, and for each edge in the boundary of $G_{10}$ we let $i$ be the integer for which the edge equals $e_{i}, 1 \leq i \leq n$, and associate the edge with the plane $h_{i}$. This information corresponds to the start of the growth process, i. e. when $k$ equals zero and no growth has taken place yet. Next, given $k, 0 \leq k<q$, we assume that a positive number (zero when $k$ equals zero) $z_{k}$, a positive integer $p_{k}$, simple polygons $G_{j k}, j=1, \ldots, p_{k}$, in the plane $z=z_{k}$, and regions (line segments when $k$ equals zero) $R_{i k} \subset \hat{h}_{i}, i=1, \ldots, n$, have been identified such that the perpendicular projection onto the $x-y$ plane of $G_{j k}, j=1, \ldots, p_{k}$, and $R_{i k}, i=1, \ldots, n$, is a collection of simple polygons (some line segments when $k$ equals zero) having pair-wise disjoint relative interiors, the union of which is $G$, and such that for each $j, j=1, \ldots, p_{k}$, every edge in the boundary of $G_{j k}$ is contained in and associated with one of the planes $h_{i}, i=1, \ldots, n$. We then show how to obtain from the information at the $k^{t h}$ level a positive number $z_{k+1}>z_{k}$, a positive integer (zero when $k+1$ equals $q$ ) $p_{k+1}$, simple polygons (empty collection when $k+1$ equals $q$ ) $G_{j, k+1}, j=1, \ldots, p_{k+1}$, in the plane $z=z_{k+1}$, and regions $R_{i, k+1}, R_{i k} \subseteq R_{i, k+1} \subset \hat{h}_{i}, i=1, \ldots, n$, such that the perpendicular projection onto the $x-y$ plane of $G_{j, k+1}, j=1, \ldots, p_{k+1}$, and $R_{i, k+1}, i=1, \ldots, n$, is a collection of simple polygons having pair-wise disjoint relative interiors, the union of which is $G$, and show how to associate each edge in the boundary of each polygon $G_{j, k+1}$, $j=1, \ldots, p_{k+1}$, with one of the planes $h_{i}, i=1, \ldots, n$, in which the edge is contained. We notice that $q$ is selected so that $p_{q}$ equals zero, the collection of simple polygons at the $q^{\text {th }}$ level is empty, and for each $i, i=1, \ldots, n, R_{i}$ equals $R_{i q}$.

In order to continue we need more notation. Given integers $l, m, 1 \leq l, m \leq n$, and assuming the planes $h_{l}$ and $h_{m}$ are not identical or parallel, we let $L_{l m}=L_{m l}$ be the straight line that is the intersection of the planes $h_{l}$ and $h_{m}$. Given $j, 1 \leq j \leq p_{k}$, and $v$, w, edges of the boundary of the polygon $G_{j k}$ such that $v$ and $w$ are adjacent to each other, we let $h_{r}, h_{s}, 1 \leq r, s \leq n$, be the planes associated with $v$ and $w$, respectively. Given $z^{\prime}, z^{\prime}>z_{k}$, we denote by $L_{r s}\left(z^{\prime}\right)$ the closed line segment in $L_{r s}$ between the planes $z=z_{k}$ and $z=z^{\prime}$. We say that $L_{r s}\left(z^{\prime}\right)$ is feasible in $G_{j k}$ if its perpendicular projection onto the plane $z=z_{k}$ lies entirely in $G_{j k}$. Given $j, 1 \leq j \leq p_{k}$, and $u$, an edge of the boundary of the polygon $G_{j k}$ associated with the plane $h_{m}, 1 \leq m \leq n$, we let $v, w$ be edges of the boundary of the polygon $G_{j k}$ such that $v$ and $w$ are adjacent to $u, v \neq w$, and let $h_{r}, h_{s}, 1 \leq r, s \leq n$, be the planes associated with $v$ and $w$, respectively. Given $z^{\prime}, z^{\prime}>z_{k}$, we say that a region can grow from $u$ to a height of $z^{\prime}$ if the lines $L_{m r}$ and $L_{m s}$ do not have a point in common whose $z$-coordinate is between $z_{k}$ and $z^{\prime}$. If a region can grow from $u$ to a height of $z^{\prime}$, we denote by $T\left(u, z^{\prime}\right)$ the closure of the region in $\hat{h}_{m}$ between the lines $L_{m r}$ and $L_{m s}$ and the planes $z=z_{k}$ and $z=z^{\prime}$. We say $T\left(u, z^{\prime}\right)$ is feasible in $G_{j k}$ if its perpendicular projection onto the plane 
$z=z_{k}$ lies entirely in $G_{j k}$. Finally, given $j, 1 \leq j \leq p_{k}$, and $u, v, w$, edges of the boundary of the polygon $G_{j k}$ such that $v$ and $w$ are adjacent to each other and $u \neq v, u \neq w$, we let $h_{m}, h_{r}, h_{s}, 1 \leq m, r, s \leq n$, be the planes associated with $u, v$ and $w$, respectively. Given $z^{\prime}, z^{\prime}>z_{k}$, we say that $\left\langle m, r, s>\right.$ is attainable in $G_{j k}$ at a height of $z^{\prime}$ if a region can grow from $u$ to a height of $z^{\prime}$ and numbers $x^{\prime}, y^{\prime}$ exist such that $L_{r s}$ intersects $\hat{h}_{m}$ at $\left(x^{\prime}, y^{\prime}, z^{\prime}\right)$ and $\left(x^{\prime}, y^{\prime}, z^{\prime}\right) \in T\left(u, z^{\prime}\right)$. We say that $\langle m, r, s\rangle$ is feasible in $G_{j k}$ at a height of $z^{\prime}$ if $\langle m, r, s\rangle$ is attainable in $G_{j k}$ at a height of $z^{\prime}$, and $L_{r s}\left(z^{\prime}\right)$ and $T\left(u, z^{\prime}\right)$ are feasible in $G_{j k}$.

With this notation we can now show how to obtain the desired information at the $(k+1)^{t h}$ level. We compute

$$
\begin{gathered}
z_{k+1}=\min \left\{z^{\prime}: \text { for some } m, r, s, j, 1 \leq m, r, s \leq n, 1 \leq j \leq p_{k},\right. \\
\left.<m, r, s>\text { is feasible in } G_{j k} \text { at a height of } z^{\prime}\right\} .
\end{gathered}
$$

For each $i, i=1, \ldots, n$, we let $R_{i, k+1}$ be the union of $R_{i k}$ with all regions of the form $T\left(u, z_{k+1}\right)$, where for some $j, 1 \leq j \leq p_{k}, u$ is an edge of $G_{j k}$ associated with the plane $h_{i}$. We let $F$ be the set of points in the plane $z=z_{k+1}$ that belong to the union of the regions $R_{i, k+1}, i=1, \ldots, n$. We let $p_{k+1}$ be the number of simple polygons that $F$ encloses, and if $p_{k+1}$ is positive we denote by $G_{j, k+1}, j=1, \ldots, p_{k+1}$, these polygons. Given $j, 1 \leq j \leq p_{k+1}$, let $u$ be an edge of the boundary of $G_{j, k+1}$. Then, from the definition of $F$, for some positive integer $n^{\prime}$ and some function $f$ from $\left\{1, \ldots, n^{\prime}\right\}$ into $\{1, \ldots, n\}$, there exist line segments $u_{f\left(i^{\prime}\right)}, i^{\prime}=1, \ldots, n^{\prime}$, having pair-wise disjoint relative interiors, such that $u$ is their union, and for each $i^{\prime}, i^{\prime}=1, \ldots, n^{\prime}, u_{f\left(i^{\prime}\right)} \subset R_{f\left(i^{\prime}\right), k+1}$. Among the planes $h_{f\left(i^{\prime}\right)}, i^{\prime}=1, \ldots, n^{\prime}$, we select one using some type of tie-breaking procedure (discussed below) and associate $u$ with this plane. If $p_{k+1}$ equals zero then $k+1$ equals $q$. Figure 4 illustrates a view from above of the portion of the growth surface for a simple polygon $G$ which is obtained during the transition in the growth process from level 0 to level 1 for the case in which all slopes slant toward the polygon at the same angle. Here $\langle 4,1,2\rangle$ is feasible in $G_{10}=G$ at a height of $z_{1}$, and the polygons $G_{11}$ and $G_{21}$ are contained in the plane $z=z_{1}$.

Next, we show that the growth surface as defined above is well-defined. In order to do this we show that $z_{k+1}$ and $q$ are well-defined. In the following lemma the existence of triplets of integers that are attainable in $G_{j k}$ at some height for each $j, j=1, \ldots, p_{k}$, is established.

Lemma 1. Given $j, 1 \leq j \leq p_{k}$, there exist $m, r, s, z^{\prime}, 1 \leq m, r, s \leq n, z^{\prime}>z_{k}$, such that $<m, r, s\rangle$ is attainable in $G_{j k}$ at a height of $z^{\prime}$.

Proof. For some positive integer $n^{\prime}$, let $e_{i}^{\prime}, i=1, \ldots, n^{\prime}$, be the edges of the boundary of 


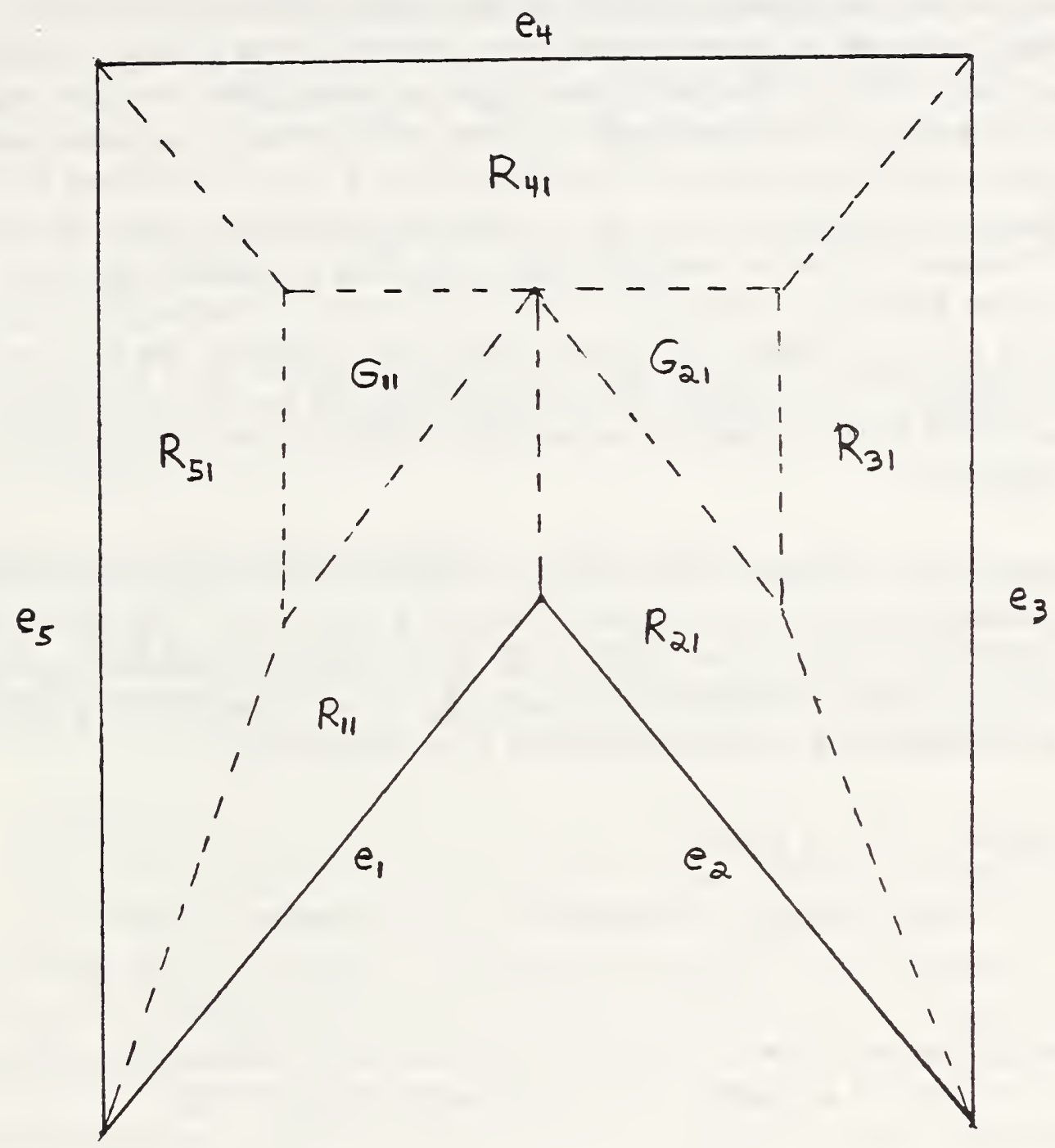

Figure 4: View from above of portion of growth surface for a simple polygon obtained during transition in growth process from level 0 to level 1 for the case in which all slopes slant toward the polygon at the same angle. 
$G_{j k}$. Let $\epsilon>0$ be a number such that for each $i, i=1, \ldots, n^{\prime}$, a region can grow from $e_{i}^{\prime}$ to a height of $z_{k}+2 \epsilon$, and the regions $T\left(e_{i}^{\prime}, z_{k}+\epsilon\right), i=1, \ldots, n^{\prime}$, are feasible in $G_{j k}$ and have pair-wise disjoint relative interiors. Let $G^{\prime \prime}$ be the simple polygon in the plane $z=z_{k}+\epsilon$ whose boundary is the set of points in that plane that belong to at least one region of the form $T\left(e_{i}^{\prime}, z_{k}+\epsilon\right), 1 \leq i \leq n^{\prime}$. Then $G^{\prime \prime}$ has $n^{\prime}$ edges in its boundary, and these edges can be denoted by $e_{i}^{\prime \prime}, i=1, \ldots, n^{\prime}$, in such a way that for each $i, i=1, \ldots, n^{\prime}, e_{i}^{\prime \prime} \subset T\left(e_{i}^{\prime}, z_{k}+\epsilon\right)$. Since the perpendicular projection of $G^{\prime \prime}$ onto the plane $z=z_{k}$ is contained in the relative interior of $G_{j k}$ and for each $i, i=1, \ldots, n^{\prime}, e_{i}^{\prime}$ and $e_{i}^{\prime \prime}$ are parallel, it follows that for some $i$, $1 \leq i \leq n^{\prime}$, the length of $e_{i}^{\prime \prime}$ is less than the length of $e_{i}^{\prime}$. This implies that for some $z^{\prime}, z^{\prime}>z_{k}$, a region can grow from $e_{i}^{\prime}$ to a height of $z^{\prime}$ but not above it. Assuming that $e_{i}^{\prime}$ is the edge of the boundary of $G_{j k}$ for which this happens with the smallest $z^{\prime}$, we let $v, w, v \neq w$, be edges of the boundary of $G_{j k}$ that are adjacent to $e_{i}^{\prime}$, and let $h_{m}, h_{r}, h_{s}, 1 \leq m, r, s \leq n$, be the planes associated with $v, e_{i}^{\prime}$ and $w$, respectively. It follows that $\langle m, r, s\rangle$ is attainable in $G_{j k}$ at a height of $z^{\prime}$.

The following lemma together with Lemma 1 establishes the existence of triplets of integers that are feasible in $G_{j k}$ at some height for each $j, j=1, \ldots, p_{k}$. This shows that $z_{k+1}$ is well-defined. The lemma also shows that in order to obtain $z_{k+1}$ it suffices to minimize over all triplets of integers that are attainable in some $G_{j k}$ at some height, $1 \leq j \leq p_{k}$. The last observation is of importance in the development of our algorithm.

Lemma 2. Given $j, 1 \leq j \leq p_{k}$, let

$$
\begin{aligned}
z_{j, k+1}^{\prime} & =\min \left\{z^{\prime}: \text { for some } m^{\prime}, r^{\prime}, s^{\prime}, 1 \leq m^{\prime}, r^{\prime}, s^{\prime} \leq n,\right. \\
& \left.<m^{\prime}, r^{\prime}, s^{\prime}>\text { is attainable in } G_{j k} \text { at a height of } z^{\prime}\right\} .
\end{aligned}
$$

Let $m, r, s$ be integers, $1 \leq m, r, s \leq n$, such that $\langle m, r, s\rangle$ is attainable in $G_{j k}$ at a height of $z_{j, k+1}^{\prime}$. Then $\langle m, r, s\rangle$ is feasible in $G_{j k}$ at a height of $z_{j, k+1}^{\prime}$. Thus

$$
z_{k+1}=\min \left\{z_{j, k+1}^{\prime}: j=1, \ldots, p_{k}\right\} .
$$

Proof. By Lemma $1, z_{j, k+1}^{\prime}$ is well-defined. Let $u$ be the edge of the boundary of $G_{j k}$ that is associated with $h_{m}$. We need to show that $T\left(u, z_{j, k+1}^{\prime}\right)$ and $L_{r s}\left(z_{j, k+1}^{\prime}\right)$ are feasible in $G_{j k}$. For simplicity we only do this for $L_{r s}\left(z_{j, k+1}^{\prime}\right)$. Assuming that $L_{r s}\left(z_{j, k+1}^{\prime}\right)$ is not feasible in $G_{j k}$, we may think of the perpendicular projection of $L_{r s}\left(z_{j, k+1}^{\prime}\right)$ onto the plane $z=z_{k}$ as originating at a vertex of $G_{j k}$, extending through the relative interior of $G_{j k}$, crossing the boundary of 
$G_{j k}$, and extending beyond. But from the definition of $z_{j, k+1}^{\prime}$, a region can grow from each edge of the boundary of $G_{j k}$ to a height of $z_{j, k+1}^{\prime}$. Thus, the perpendicular projection of $L_{r s}\left(z_{j, k+1}^{\prime}\right)$ onto the plane $z=z_{k}$ can cross the boundary of $G_{j k}$ as described only if for some edge $v$ in the boundary of $G_{j k}$ and some number $\hat{z}, \hat{z}>z_{k}, L_{r s}(\hat{z})$ and $T(v, \hat{z})$ have a point in common with $z$-coordinate $\hat{z}$ and $L_{r s}(\hat{z})$ is feasible in $G_{j k}$. But this implies $\hat{z}<z_{j, k+1}^{\prime}$ which then contradicts the definition of $z_{j, k+1}^{\prime}$. This completes the proof of the lemma.

In order to show that $q$ is well-defined we need the following two lemmas. The proofs extend to the case in which $G$ is multiply-connected.

Lemma 3. Let $k$ be a positive integer such that the growth surface has reached the $k^{\text {th }}$ level. For each $i, i=1, \ldots, n, R_{i k}$ and therefore its perpendicular projection onto the $x-y$ plane is a polygon.

Proof. Follows directly from the definition of $R_{i k}, i=1, \ldots, n$.

Lemma 4. Let $k$ be a positive integer such that the growth surface has reached the $k^{\text {th }}$ level. Given integers $m, r, s, 1 \leq m, r, s \leq n$, at most one integer $l, 0<l \leq k$, can exist such that for some $j, 1 \leq j \leq p_{l-1},\langle m, r, s\rangle$ is feasible in $G_{j, l-1}$ at a height of $z_{l}$.

Proof. We assume that for some $l, 0<l \leq k$, and some $j, 1 \leq j \leq p_{l-1},\langle m, r, s\rangle$ is feasible in $G_{j, l-1}$ at a height of $z_{l}$, and that this is the smallest $l$ for which this happens. It follows that either $L_{r s}$ intersects $h_{m}$ at one point or $L_{r s}$ is contained in $h_{m}$. In the first case the lemma follows trivially. In the second case let $l^{\prime}$ be an integer, $l<l^{\prime} \leq k$, such that for some $j^{\prime}, 1 \leq j^{\prime} \leq p_{l^{\prime}-1},<m, r, s>$ is feasible in $G_{j^{\prime}, l^{\prime}-1}$ at a height of $z_{l^{\prime}}$. It follows that a portion of $L_{r s}$ right below the plane $z=z_{l}$ and another one right below the plane $z=z_{l}$, belong to the growth surface. Since $L_{r s}$ intersects $\hat{h}_{m}$ at $z=z_{l}$ which is between the two aforementioned portions of $L_{r s}$, and at $z=z_{l^{\prime}}$ which is above the second portion, it follows from the fact that $R_{r l^{\prime}}$ and $R_{s l^{\prime}}$ are polygons (Lemma 3 ) that $R_{m l^{\prime}}$ is not connected, a contradiction to the fact that $R_{m l^{\prime}}$ is a polygon (Lemma 3 ). The lemma follows.

Finally, the following lemma shows that $q$ is well-defined.

Lemma 5. There exists a positive integer $q$ such that $p_{q}$ equals zero, and for each $k$, $0 \leq k<q, p_{k}$ is positive. 
Proof. Let $k$ be a non-negative integer such that the growth surface has reached the $k^{\text {th }}$ level. If $p_{k}$ is positive it follows from Lemma 1 and Lemma 2 that integers $m, r, s, j$ exist, $1 \leq m, r, s \leq n, 1 \leq j \leq p_{k}$, such that $\langle m, r, s\rangle$ is feasible in $G_{j k}$ at a height of $z_{k+1}$. But this can only happen a finite number of times from Lemma 4 and the fact that the number of possible permutations of $n$ things 3 at a time is finite. Thus, for some $q>0, p_{q}$ must equal zero and this is the first $q$ for which this happens.

In the above definition of the growth surface for the slopes at the boundary of a polygon, a tie-breaking procedure was mentioned. Given a positive integer $k$ such that the growth surface has reached the $k^{\text {th }}$ level with $p_{k} \neq 0$, such a procedure is required if for some $j$, $1 \leq j \leq p_{k}$, the boundary of $G_{j k}$ contains an edge $u$ such that for some integer $n^{\prime} \geq 2$ and some function $f$ from $\left\{1, \ldots, n^{\prime}\right\}$ into $\{1, \ldots, n\}$, there exist line segments $u_{f\left(i^{\prime}\right)}, i^{\prime}=1, \ldots, n^{\prime}$, having pair-wise disjoint relative interiors with the properties that $u$ equals their union and for each $i^{\prime}, i^{\prime}=1, \ldots, n^{\prime}, u_{f\left(i^{\prime}\right)} \subset R_{f\left(i^{\prime}\right), k}$. Whenever this happens, given $l^{\prime}, m^{\prime}, 1 \leq l^{\prime}, m^{\prime} \leq n^{\prime}$, $l^{\prime} \neq m^{\prime}$, the edges $e_{f\left(l^{\prime}\right)}, e_{f\left(m^{\prime}\right)}$ of the boundary of the polygon $G$ are either parallel or colinear and the planes $h_{f\left(l^{\prime}\right)}$ and $h_{f\left(m^{\prime}\right)}$ are identical or intersect at the line that contains the edge $u$. This is why it only makes sense to associate the same plane with all of the edges $e_{f\left(i^{\prime}\right)}$, $i^{\prime}=1, \ldots, n^{\prime}$, and for that matter with their union $u$. In keeping with the spirit of the definition of the growth surface one then selects one of the planes $h_{f\left(i^{\prime}\right)}, i^{\prime}=1, \ldots, n^{\prime}$, and associates $u$ with this plane. In order to select one of the planes a tie-breaking procedure is necessary. Accordingly, one such procedure selects the plane that slants the most toward the polygon. Another one selects the plane that slants the least.

The assumptions made about the planes $h_{i}, i=1, \ldots, n$, can be relaxed in such a way that the lemmas presented above still hold and a growth surface for the slopes at the boundary of the polygon $G$ can still be defined. Again, we consider a simple or multiply-connected polygon $G$ with $n$ vertices that is contained in the $x-y$ plane of 3 -dimensional space. We let $e_{i}, i=1, \ldots, n$, be the edges of the boundary of $G$. For each $i, i=1, \ldots, n$, we let $O_{i}$ be an open convex set in the relative interior of $G$ whose closure includes $e_{i}$, and we let $g_{i}$ be the closed half-plane in the $x-y$ plane whose relative interior contains $O_{i}$ and whose boundary contains $e_{i}$. In what follows, for each $i, i=1, \ldots, n$, given a closed half-plane $\hat{h}$ in the upper closed half-space defined by the $x-y$ plane, if $\hat{h}$ contains $e_{i}$ we denote the measure of the smaller of the two angles between $\hat{h}$ and $g_{i}$ by $\alpha_{i}(\hat{h})$. We assume that a plane $h^{\prime}$ in 3-dimensional space has been given which is not perpendicular to the $x-y$ plane and we let $G^{\prime}$ be the perpendicular projection onto $h^{\prime}$ of $G$. We let $e_{i}^{\prime}, i=1, \ldots, n$, be the edges of $G^{\prime}$ in such a way that for each $i, i=1, \ldots, n, e_{i}^{\prime}$ is the perpendicular projection 
onto $h^{\prime}$ of $e_{i}$. We let $h_{i}^{\prime}, i=1, \ldots, n$, be planes in 3 -dimensional space perpendicular to $h^{\prime}$ in such a way that for each $i, i=1, \ldots, n, e_{i}^{\prime} \subset h_{i}^{\prime}$. Clearly, for each $i, i=1, \ldots, n, e_{i} \subset h_{i}^{\prime}$. For each $i, i=1, \ldots, n$, we let $\hat{h}_{i}^{\prime}$ be the closed half-plane in $h_{i}^{\prime}$ which is the intersection of $h_{i}^{\prime}$ and the upper closed half-space defined by the $x-y$ plane. Finally, we assume that planes $h_{i}, i=1, \ldots, n$, have been chosen such that for each $i, i=1, \ldots, n, e_{i}$ is contained in $h_{i}$, and with $\hat{h}_{i}$ denoting for each $i, i=1, \ldots, n$, the closed half-plane in $h_{i}$ which is the intersection of $h_{i}$ and the upper closed half-space defined by the $x-y$ plane, we assume that for each $i, i=1, \ldots, n, \alpha_{i}\left(\hat{h}_{i}\right) \leq \alpha_{i}\left(\hat{h}_{i}^{\prime}\right)$. Under these assumptions and using arguments similar to the ones used above, it is possible to show that a growth surface for the polygon can be properly defined as above if the following condition holds: For each $k, k \geq 0$, with $p_{k} \neq 0$, and each $j, 1 \leq j \leq p_{k}$, there exists at least one edge $u$ in the boundary of $G_{j k}$ such that if $h_{l}, 1 \leq l \leq n$, is the plane associated with $u$ then $\alpha_{l}\left(\hat{h}_{l}\right)<\alpha_{l}\left(\hat{h}_{l}^{\prime}\right)$. This condition is satisfied if, for example, for at least one $l, 1 \leq l \leq n$, it is the case that $\alpha_{l}\left(\hat{h}_{l}\right)<\alpha_{l}\left(\hat{h}_{l}^{\prime}\right)$, and the tie-breaking procedure used is the one that selects the plane that slants the most toward the polygon.

Finally, we notice that the ridge of the growth surface for a polygon is a tree-like structure whose edges are all straight-line segments and that if the polygon is simple this structure consists of exactly one connected component. We also notice that the ridge of the growth surface for a multiply-connected polygon does not necessarily consist of one connected component. This is the case when for some edge in the boundary of the polygon, the region that grows from the edge does not turn out to be a simple polygon. We illustrate this situation with an example. We consider the multiply-connected polygon in Figure 5 whose boundary has $e_{i}, i=1, \ldots, 8$, as edges. Assuming this polygon is contained in the $x-y$ plane of 3 -dimensional space we let $h_{1}$ be any plane that contains $e_{1}$ and that slants toward the polygon, and for each $i, i=2, \ldots, 8$, we let $h_{i}$ be the plane that contains $e_{i}$ and that is perpendicular to the $x-y$ plane. Under these assumptions, it follows that the growth surface for the polygon is such that the perpendicular projection of $R_{1}$ onto the $x-y$ plane is the polygon itself while those of $R_{i}, i=2, \ldots, 8$, are the edges $e_{i}, i=2, \ldots, 8$, respectively. Accordingly, the ridge of the growth surface consists of two connected components, that correspond essentially to the outer and inner boundaries of the polygon, respectively, and that are separated from each other by the interior of $R_{1}$.

\section{A brute force algorithm}

In this section we present a brute force algorithm in the form of a procedure called GROSUR for computing the growth surface for the slopes at the boundary of a simple or multiplyconnected polygon. The algorithm follows closely the definition by induction of the growth 


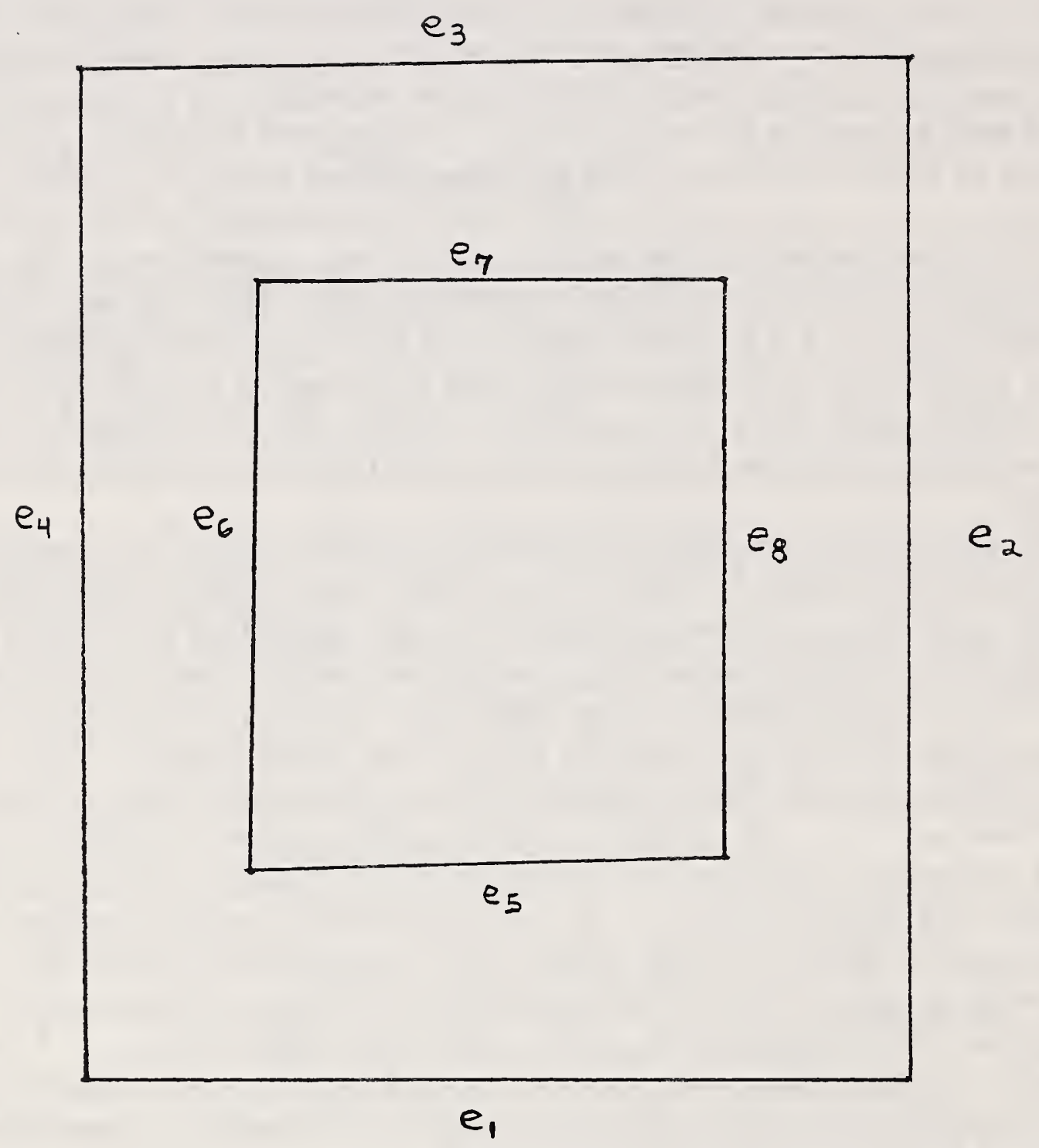

Figure 5: A multiply-connected polygon. 
surface for a polygon presented in the previous section, and its justification follows from Lemma 2 in the same section. Actually, a stronger version of Lemma 2 is required. In order to present this new version of the lemma we expand on the notion of 'attainable.' Given $j, l$, $1 \leq j, l \leq p_{k}, u$, an edge of the boundary of the polygon $G_{j k}$, and $v, w$, edges of the boundary of the polygon $G_{l k}$ such that $v$ and $w$ are adjacent to each other and $u \neq v, u \neq w$, we let $h_{m}, h_{r}, h_{s}, 1 \leq m, r, s \leq n$, be the planes associated with $u, v$, and $w$, respectively. Given $z^{\prime}, z^{\prime}>z_{k}$, we say that $\langle m, r, s\rangle$ is attainable in $G_{j k} \cup G_{l k}$ at a height of $z^{\prime}$ if a region can grow from $u$ to a height of $z^{\prime}$ and numbers $x^{\prime}, y^{\prime}$ exist such that $L_{r s}$ intersects $\hat{h}_{m}$ at $\left(x^{\prime}, y^{\prime} z^{\prime}\right)$ and $\left(x^{\prime}, y^{\prime}, z^{\prime}\right) \in T\left(u, z^{\prime}\right)$. The new version of Lemma 2, which we call Lemma 2', follows.

Lemma 2'. Let

$$
\begin{gathered}
z_{k+1}^{\prime}=\min \left\{z^{\prime}: \text { for some } m^{\prime}, r^{\prime}, s^{\prime}, j^{\prime}, l^{\prime}, 1 \leq m^{\prime}, r^{\prime}, s^{\prime} \leq n, 1 \leq j^{\prime}, l^{\prime} \leq p_{k},\right. \\
\left.<m^{\prime}, r^{\prime}, s^{\prime}>\text { is attainable in } G_{j^{\prime} k} \cup G_{l^{\prime} k} \text { at a height of } z^{\prime}\right\} .
\end{gathered}
$$

Let $m, r, s, j, l$ be integers, $1 \leq m, r, s, \leq n, 1 \leq j, l \leq p_{k}$, such that $\langle m, r, s\rangle$ is attainable in $G_{j k} \cup G_{l k}$ at a height of $z_{k+1}^{\prime}$. Then $j$ equals $l$ and $\langle m, r, s\rangle$ is feasible in $G_{j k}\left(=G_{l k}\right)$ at a height of $z_{k+1}^{\prime}$. Thus $z_{k+1}$ equals $z_{k+1}^{\prime}$.

In what follows, given integers $m, r, s, 1 \leq m, r, s \leq n$, and a number $z^{\prime}, z^{\prime}>z_{k}$, we say that $\langle m, r, s\rangle$ is attainable in $F \equiv \cup_{j^{\prime}=1}^{p_{k}} G_{j^{\prime} k}$ at a height of $z^{\prime}$ if for integers $j, l$, $1 \leq j, l \leq p_{k},\langle m, r, s\rangle$ is attainable in $G_{j k} \cup G_{l k}$ at a height of $z^{\prime}$. This definition and Lemma 2' allow the computation of $z_{k+1}$ when only information about the edges of the boundaries of the polygons $G_{j k}, j=1, \ldots, p_{k}$, is available.

Let $G, n, e_{i}, h_{i}, q, z_{k}, p_{k}, R_{i k}, G_{j k}, k=0, \ldots, q, i=1, \ldots, n, j=1, \ldots, p_{k}$, be as defined in the previous section. Given $k, j, 0 \leq k<q, 1 \leq j \leq p_{k}$, $u$, an edge of the boundary of $G_{j k}$, and $z^{\prime}, z^{\prime}>z_{k}$, such that a region can grow from $u$ to a height of $z^{\prime}$, we let $T\left(u, z^{\prime}\right)$ be also as defined in the previous section. In the following we list and describe variables and procedures used in procedure GROSUR. However, in order to do so an observation and some notation are necessary. First the observation. We notice that each execution of line 4 of GROSUR (listed below) corresponds to a growth level $k$, starting with $k$ equal to zero, and that no variable corresponding to $k$ is explicitly used in the procedure. Neither are variables that would correspond to $p_{k}$ and $G_{j k}, k=0, \ldots, q, j=1, \ldots, p_{k}$. Thus, in what follows, whenever we say 'the polygons at the current level' we will mean that GROSUR is currently being executed, that at the present moment $k$ is implicitly defined and set equal to some non-negative integer, and that the polygons at the current level are the polygons 
$G_{j k}, j=1, \ldots, p_{k}$, which are also implicitly defined. All of this is in accordance with the observation after Lemma 2 ' that essentially states that in order to compute $z_{k+1}$, complete information about the polygons $G_{j k}, j=1, \ldots, p_{k}$, is not necessary and that in fact certain information about the edges of the boundaries of the polygons suffices. Finally the notation. Let $e$ be an edge of the boundary of a polygon. Let $O$ be a closed rectangle contained in the polygon with $e$ as one of its sides. Let $Q_{L}$ and $Q_{R}$ be the endpoints of $e$. We say that $Q_{L}$ is the left endpoint of $e$ and that $Q_{R}$ is the right endpoint of $e$ if in a counterclockwise direction around the interior of $O, Q_{R}$ is the first vertex of $O$ that appears after $Q_{L}$ in the boundary of $O$. The descriptions of variables and procedures follow.

$R$ : A function whose domain is the set $\{1, \ldots, n\}$. As an input variable for GROSUR, for each $i, i=1, \ldots, n, R(i)$ equals $e_{i}$. At the start of each execution of line 4 of procedure GROSUR, for each $i, i=1, \ldots, n, R(i)$ equals the $i^{\text {th }}$ growth region up to the current level. As an output variable for GROSUR, for each $i, i=1, \ldots, n, R(i)$ equals the $i^{\text {th }}$ growth region in the growth surface for the polygon.

$H$ : A function whose domain is the set $\{1, \ldots, n\}$. At all times, for each $i, i=1, \ldots, n, H(i)$ equals plane $h_{i}$.

$p$ : A non-negative integer. As an input variable for GROSUR, $p$ equals $n$, and at the start of each execution of line 4 of GROSUR equals the total number of edges in the boundaries of the polygons at the current level.

$U$ : A function whose domain is the set $\{1, \ldots, p\}$. As an input variable for GROSUR, for each $j, j=1, \ldots, p, U(j)$ equals $e_{j}$. At the start of each execution of line 4 of GROSUR with $p \neq 0$, for each $j, j=1, \ldots, p, U(j)$ equals the $j^{\text {th }}$ edge in the boundaries of the polygons at the current level.

$N$ : A function whose domain is the set $\{1, \ldots, p\}$. As an input variable for GROSUR and at the start of each execution of line 4 of GROSUR with $p \neq 0$, for each $j, j=1, \ldots, p, N(j)$ equals the edge adjacent to $U(j)$ in the boundary of the polygon that contains $U(j)$, the left endpoint of which is the right endpoint of $U(j)$.

$P$ : A function whose domain is the set $\{1, \ldots, p\}$. As an input variable for GROSUR and at the start of each execution of line 4 of GROSUR with $p \neq 0$, for each $j, j=1, \ldots, p, P(j)$ equals the edge adjacent to $U(j)$ in the boundary of the polygon that contains $U(j)$, the 
right endpoint of which is the left endpoint of $U(j)$.

$\hat{z}$ : A non-negative number. At the start of each execution of line 4 of GROSUR, $\hat{z}$ equals the elevation of the current level.

$r$ : A function whose domain is the set $\{1, \ldots, p\}$. At the start of each execution of line 4 of GROSUR with $p \neq 0$, for each $j, j=1, \ldots, p, r(j)$ equals the number of line segments having pair-wise disjoint relative interiors into which $U(j)$ can be partitioned with the property that given $l, 1 \leq l \leq r(j)$, there exists $i, 1 \leq i \leq n$, such that the $l^{\text {th }}$ segment in the partition of $U(j)$ is contained in $R(i)$.

$V$ : A function whose domain consists of pairs of positive integers of the form $\{l, j\}$, where $1 \leq j \leq p$, and $1 \leq l \leq r(j)$. At the start of each execution of line 4 of GROSUR with $p \neq 0$, for each $j, j=1, \ldots, p, V(1, j), \ldots, V(r(j), j)$ are line segments having pair-wise disjoint relative interiors with the property that $U(j)$ equals $\cup_{l=1}^{r(j)} V(l, j)$, and that given $l, 1 \leq l \leq r(j)$, there exists $i, 1 \leq i \leq n$, such that $V(l, j) \subseteq R(i)$.

$A$ : A function whose domain consists of line segments computed in GROSUR and whose range is the set $\{1, \ldots, n\}$. At the start of each execution of line 4 of GROSUR with $p \neq 0$, for each $j, j=1, \ldots, p$, and each $l, 1 \leq l \leq r(j), A(V(l, j))$ equals the integer $i, 1 \leq i \leq n$, for which $V(l, j) \subseteq R(i)$.

$\operatorname{TIEBRK}(H, A, V, r, j, l)$ : Given $j, 1 \leq j \leq p$, and using some type of tie-breaking criterion, procedure TIEBRK selects one of the planes $H(A(V(1, j))), \ldots, H(A(V(r(j), j)))$. The integer $l, 1 \leq l \leq r(j)$, is the one for which $H(A(V(l, j)))$ is the selected plane.

$\operatorname{MINATT}(\hat{z}, H, U, N, P, p, A)$ : Assuming that the edges $U(j), j=1, \ldots, p$, are the edges of the boundaries of the polygons at the current level, say level $k, k \geq 0$, with $z_{k}=\hat{z}$, and using functions $H, N, P, A$ for any other required information, procedure MINATT computes $\hat{z}=z_{k+1}$ as in Lemma 2' above.

TGROWT $(\hat{z}, H, U, N, P, j, A, T)$ : Given $j, 1 \leq j \leq p$, assuming that $U(j)$ is an edge of the boundary of a polygon at the current level and that a region can grow from $U(j)$ to a height of $\hat{z}$, and using functions $H, N, P, A$ for any required information, procedure TGROWT computes $T=T(U(j), \hat{z})$. 
$\operatorname{BEDGES}(R, \hat{z}, U, N, P, p, V, r, A)$ : Through an analysis for each $j, j=1, \ldots, p$, of the set of points in $R(A(U(j)))$ with $z$-coordinate equal to $\hat{z}$, and using functions $N, P$ for any other required information, procedure BEDGES computes $p, U(j), N(j), P(j), r(j), V(l, j)$, $A(V(l, j)), j=1, \ldots, p, l=1, \ldots, r(j)$, at the next level.

Procedure GROSUR now follows.

procedure GROSUR $(R, H, p, U, N, P)$

$$
\text { begin }
$$

1. $\hat{z}:=0$

2. for $j:=1$ until $p$ do

begin

3. $\quad r(j):=1 ; V(1, j):=U(j) ; A(V(1, j)):=j$

end

4. while $(p \neq 0)$ do

begin

5. for $j:=1$ until $p$ do

begin

6. $\quad \operatorname{TIEBRK}(H, A, V, r, j, l)$;

7. $\quad A(U(j)):=A(V(l, j))$

$$
\text { end }
$$

8. $\quad \operatorname{MinATT}(\hat{z}, H, U, N, P, p, A)$;

9. for $j:=1$ until $p$ do

$$
\text { begin }
$$

10.

11.

$$
\begin{aligned}
& \text { TGROWT }(\hat{z}, H, U, N, P, j, A, T) \\
& R(A(U(j))):=R(A(U(j))) \cup T
\end{aligned}
$$

end

12. $\operatorname{BEDGES}(R, \hat{z}, U, N, P, p, V, r, A)$

end

end

\section{Summary}

We have defined inductively the growth surface for the slopes at the boundary of a simple or multiply-connected polygon. We have also presented a brute force algorithm for computing it 
which is essentially a restatement of its definition. Finally, we report that an implementation of the algorithm in Fortran has been completed at the National Institute of Standards and Technology.

\section{Acknowledgments}

The author gratefully acknowledges Christoph Witzgall for his encouragement during the preparation of this paper.

\section{References}

[1] D. T. Lee, Medial axis transformation of a planar shape, IEEE Trans. Patt. Anal. Mach. Intell. PAMI-4 (1982), 363-369.

[2] F. P. Preparata, The medial axis of a simple polygon, Proc. $6^{\text {th }}$ Ann. Internat. Symp. Math. Found. Computer Sci. (1977), 443-450. 

